\title{
Nanocides: smart delivery system in agriculture and horticultural crops
}

\section{Opinion}

The word "nano" comes from the Greek for "dwarf". A nanometer is a thousandth of a thousandth of a thousandth of a meter (10-9 m). One nanometer is about 60,000 times smaller than a human hair in diameter or the size of a virus, a typical sheet of paper is about 100,000 $\mathrm{nm}$ thick, a red blood cell is about 2,000 to $5,000 \mathrm{~nm}$ in size, and the diameter of DNA is in the range of $2.5 \mathrm{~nm}$. Therefore, nanotechnology deals with matter that ranges from one-half the diameter of DNA up to $1 / 20$ the size of a red blood cell. Further, it is interesting to note that nonmaterials are so small, even bacteria would need a microscope to see them. Nanoparticles are generally accepted as those with a particle size below 100 nanometers where unique phenomena enable novel applications and benefits. Nanomaterials on which most of the research has been carried out are normally powders composed of nanoparticles which exhibit properties that are different from powders of the same chemical composition, but with much larger particles. The current global population is nearly 6billion with $50 \%$ living in Asia. A large proportion of those living in developing countries face daily food shortages as a result of environmental impacts or political instability, while in the developed world there is a food surplus. The challenge is how to feed the growing population by producing more on a stagnant or shrinking landscape; with lesser input costs and with lesser hazards to the eco-system. Another adjunct to this problem is how to add to the income of agricultural producers so as to sustain their motivation to grow crops. Nanotechnology has answers to many of these challenges. In agriculture, some of the world's largest makers of pesticides, fertilizers, and other farm inputs and technologies are betting on nanotechnology to bring unprecedented precision to crop and livestock production. Potential applications of nanotechnology in crop protection include controlled release of encapsulated pesticide, fertilizer and other agrochemicals in protection against pests and pathogens, early detection of plant disease and pollutants including pesticide residues by using nanosensors. The potential applications of nanomaterials in crop protection, helps in the development of efficient and potential approaches for the management of plant pathogens. Nanotechnology has emerged as one of the most innovative scientific field in agriculture.

It provides opportunity to develop improved systems for monitoring environmental conditions and delivering nutrients or pesticides as appropriate, improve our understanding of the biology of different crops and thus potentially enhance yields or nutritional values. Precision farming- Nanotechnology application here makes farming more targeted and scientific. Precision farming makes use of computers, global satellite positioning systems, and remote sensing devices to measure various parameters. Accurate information through applications of Nanotechnology for real time monitoring of soil conditions, environmental changes and diseases and plant health issues. Nanoscale devices with novel properties could be used to make agricultural systems "smart". For example, devices could be used to identify plant health issues before these become visible to
Volume 6 Issue 6 - 2017

\author{
Touseef Hussain \\ Division of Plant Pathology, ICAR-Indian Agricultural Research \\ Institute
}

Correspondence: Touseef Hussain, Division of Plant Pathology, ICAR-Indian Agricultural Research Institute, New Delhi-I I00 I2, Email Hussaintouseef@yahoo.co.in

Received: March 0I, 2017 | Published: March 27, 2017

the farmer. Such devices may be capable of responding to different situations by taking appropriate remedial action. If not, they will alert the farmer to the problems. In this way, smart devices will act as both a preventive and an early warning system. Such devices could be used to deliver chemicals in a controlled and targeted manner in the same way as nanomedicine has implications for drug delivery in humans. Nanotechnology has the potential to revolutionize the agricultural and food industry with new tools for the molecular treatment of diseases, rapid disease detection, enhancing the ability of plants to absorb nutrients etc. Smart sensors and smart delivery systems will help the agricultural industry combat viruses and other crop pathogens. In the near future nanostructured catalysts will be available which will increase the efficiency of pesticides and herbicides, allowing lower doses to be used. However, currently there is a limited understanding of human health and safety risks associated with this technology. The potential application of nanocides in different agricultural applications needs further research investigation with respect to synthesis, toxicology and its effective application at field level. Nanotechnology has got applicability in various fields, but research and development is still at bench-top scale. Great efforts are required in commercialization of nanocides for agricultural applications, which requires proper protection needs, testing priorities, risk assessment and regulatory guidance at global level. Controlled use of the technology will open opportunities for developing new materials and methods that will enhance our ability to develop faster, more reliable and more sensitive analytical systems. Overall the scenario presents us with the view that nanotechnology is here to stay.

\section{Acknowledgements}

None.

\section{Conflict of interest}

The author declares no conflict of interest. 